\title{
The functional significance of the skeletal muscle clock: lessons from Bmal1 knockout models
}

\author{
Stefano Schiaffino ${ }^{1 *}$ (D) Bert Blaauw $w^{1,2}$ and Kenneth A. Dyar ${ }^{3}$
}

\begin{abstract}
The circadian oscillations of muscle genes are controlled either directly by the intrinsic muscle clock or by extrinsic factors, such as feeding, hormonal signals, or neural influences, which are in turn regulated by the central pacemaker, the suprachiasmatic nucleus of the hypothalamus. A unique feature of circadian rhythms in skeletal muscle is motor neuron-dependent contractile activity, which can affect the oscillation of a number of muscle genes independently of the muscle clock. The role of the intrinsic muscle clock has been investigated using different Bmal1 knockout $(\mathrm{KO})$ models. A comparative analysis of these models reveals that the dramatic muscle wasting and premature aging caused by global conventional $\mathrm{KO}$ are not present in muscle-specific Bmal1 $\mathrm{KO}$ or in global Bmal1 KO induced in the adult, therefore must reflect the loss of Bmal1 function during development in non-muscle tissues. On the other hand, muscle-specific Bmal1 knockout causes impaired muscle glucose uptake and metabolism, supporting a major role of the muscle clock in anticipating the sleep-to-wake transition, when glucose becomes the predominant fuel for the skeletal muscle.
\end{abstract}

Keywords: Skeletal muscle, Circadian rhythms, Muscle clock, Bmal1 knockout, Muscle denervation, Glucose uptake, Glucose metabolism

\section{Background}

All tissues of the body display circadian oscillations in gene expression involving both the core clock genes, which are common to all tissues, and a large number of other genes, most of which are tissue-specific [1]. The core clock system consists of a transcriptional/ translational feedback loop whereby a complex of two basic helix-loop-helix-PAS domain-containing transcription factors, BMAL1 and CLOCK, induces the expression of Per and Cry genes, whose products repress the transcription of Bmal1 and Clock, thus inhibiting their own transcription (Fig. 1a) [2]. Bmal1 expression is controlled by additional factors, RORs and REV-ERBs, whose transcription is also regulated by the BMAL1-CLOCK complex, and is further modulated by post-transcriptional changes, such as casein kinase (CK)2-mediated phosphorylation [3]. Most core

\footnotetext{
* Correspondence: stefano.schiaffino@unipd.it

${ }^{1}$ Venetian Institute of Molecular Medicine (VIMM), Via Orus 2, 35129 Padova, Italy

Full list of author information is available at the end of the article
}

clock genes show functional redundancy due to the presence of variants, such as Per1 and Per2, or Cry1 and Cry 2 genes, so that double knockouts are required to disrupt clock function; NPAS2 can substitute for CLOCK as a partner for BMAL1, at least in some tissues $[2,4]$. In contrast, BMAL1 is a nonredundant clock component, and its ablation has been extensively used to determine the function of the muscle clock. The circadian oscillations of most cellular genes are controlled either directly by the intrinsic clock or by extrinsic factors, such as feeding, hormonal signals, or neural influences, which are regulated by the central pacemaker, the suprachiasmatic nucleus (SCN) of the hypothalamus. Here, we briefly review the regulation of circadian rhythms in the skeletal muscle, focusing on the role of the intrinsic muscle clock and critically considering the effects of different Bmal1 knockout (KO) models. These studies reveal that the intrinsic muscle clock is dispensable for muscle growth and does not affect aging and life span, in contrast to previous suggestions based on 


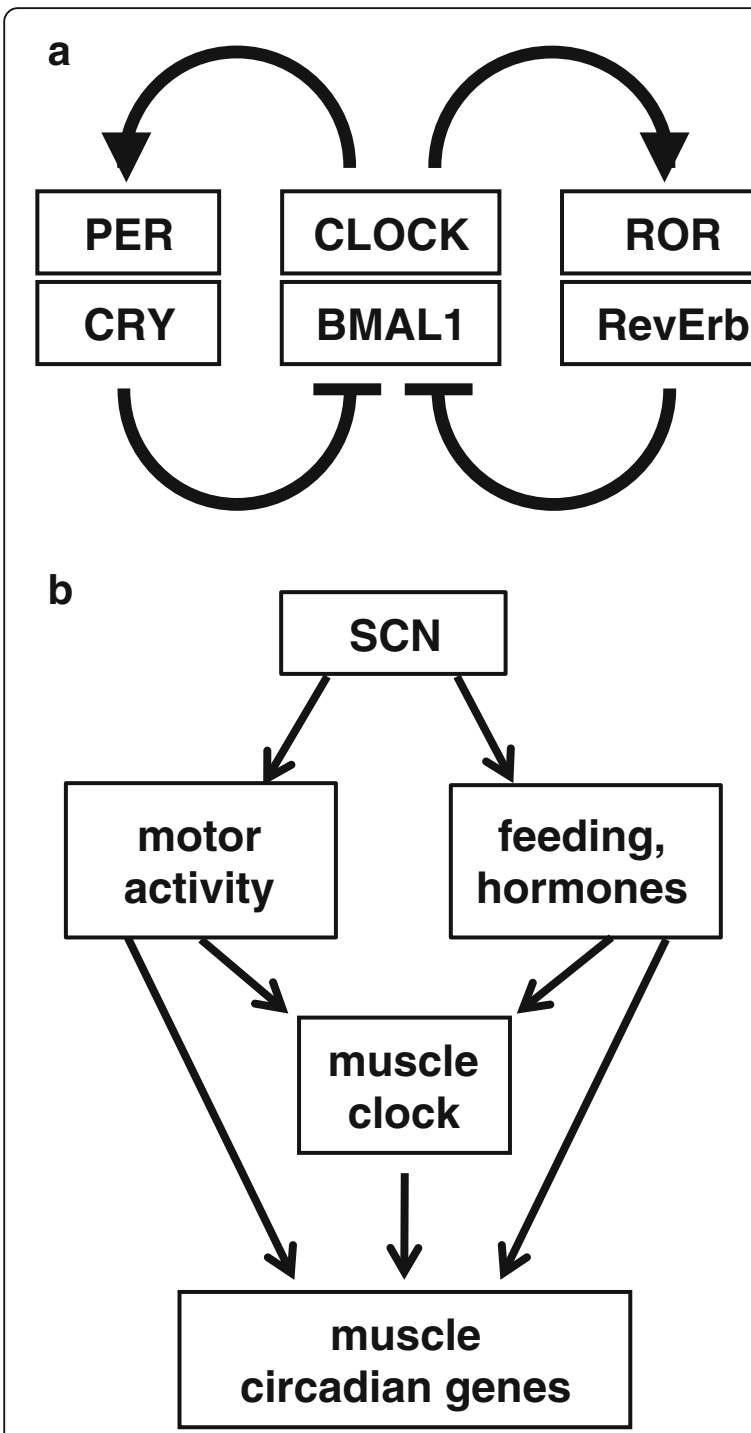

Fig. 1 Core clock mechanism and control of circadian muscle genes by intrinsic and extrinsic pathways. a Simplified scheme of the core clock mechanism. The clock consists of a stimulatory loop, with the CLOCK-BMAL1 heterodimer stimulating the transcription of Per and Cry genes, and an inhibitory feedback loop with the PER-CRY heterodimer translocating to the nucleus and repressing the transcription of the Clock and Bmall genes. An additional loop involves the ROR and RevErb factors. The different isoforms of the clock genes, including the genes coding for PER1 and PER2, CRY1 and CRY2, RORa and ROR 3 , and RevErba and RevErb $\beta$, are not indicated in the scheme. $\mathbf{b}$ The scheme illustrates how the master clock in the suprachiasmatic nuclei (SCN) of the hypothalamus controls motor activity and other systemic circadian rhythms (including feeding, hormone release, and body temperature), which in turn modulate the circadian rhythm of the muscle clock or directly dictate the oscillation of other muscle circadian genes. Modified from [7] global Bmal1 KO. On the other hand, the muscle clock has a crucial function in muscle metabolism, by anticipating the changes in glucose uptake and oxidation at the sleep-to-wake transition.

\section{Extrinsic control of circadian rhythms in the skeletal muscle: the role of motor activity}

Peripheral oscillators, including the muscle clock, are synchronized by the $\mathrm{SCN}$ through a variety of signals, including daily variations in body temperature, humoral factors, and the autonomic nervous system (reviewed in [2]). Oscillations of tissue-specific circadian genes are controlled either directly by the intrinsic peripheral clocks or indirectly by extrinsic factors (Fig. 1b). For example, inducible liver-specific repression of Bmal1 transcription abrogates the oscillation of most circadian liver genes, showing that they are directly controlled by the hepatocyte clock; however, a number of other genes continue oscillating even in the absence of a functional liver clock, showing that they are controlled by extrinsic factors [5]. Feeding has a dominant role in setting the phase of peripheral oscillators, as temporal feeding restriction, induced by offering food only during the light phase, radically changes the phase of both core clock genes and other circadian genes in peripheral tissues of mice [6], including the skeletal muscle [7]. Plasma glucocorticoid and body temperature rhythms are also involved in the synchronization of peripheral clocks [2]. Another potential extrinsic circadian signal, which is unique to skeletal muscle, is motor neuron-dependent contractile activity.

Locomotor activity has traditionally been used both in mice and in flies as a readout of the circadian timing system. One may wonder whether motor neuron activity regulates the intrinsic muscle clock and/or other muscle cycling genes. Indeed, a phase distribution analysis of the circadian muscle transcriptome revealed that the largest cluster of rhythmic genes is found at the midpoint of the active phase [8]. Exercise was found to affect both the amplitude and the phase of the circadian clock in the skeletal muscle (reviewed in $[9,10]$ ). However, interpretation of these results is complicated by the fact that exercise causes systemic effects, such as hormonal changes and increased body temperature, which are known to affect the peripheral oscillators. One-leg exercise in humans allowed for direct comparisons between active and inactive legs in the same individuals, thus excluding the potential contribution of systemic effects of exercise: the expression of core clock genes and downstream targets was modified in the exercised but not in the non-exercised contralateral leg; however, only two time points were examined in this study [11].

An alternative approach to define the role of nerve activity on circadian gene expression in the skeletal muscle 
is to compare fast-twitch, sporadically active muscles, composed by type 2 fibers, and slow-twitch, continuously active muscles, composed by type 1 fibers. Total daily activity, monitored by electromyography in rats, differs by more than 50 times between motor units composed of slow type 1 muscle fibers and motor units composed of fast-type 2B fibers [12]. The circadian expression pattern of the core clock genes was essentially the same in the two types of muscles, although most other circadian genes, including both clockdependent and clock-independent genes, were specific for each muscle [7]. A more drastic experiment is to compare completely inactive denervated muscles with normally active contralateral muscles in the same animals. Core clock genes show small but significant changes in phase, amplitude, and/or expression level in the absence of motor nerve activity [7, 13]. For example, the circadian phase of Bmall and its direct target, $\mathrm{Dbp}$, is advanced by about $3-4 \mathrm{~h}$ in denervated muscles, but both amplitude and expression levels are unchanged (Fig. 2). In addition, many other clock-dependent and clock-independent muscle circadian genes show marked alterations in absolute expression level, phase, and amplitude. Importantly, the circadian oscillation of plasma glucocorticoids or the hepatic circadian expression of clock genes are not altered by nerve section, suggesting that systemic circadian rhythms are not affected in this experimental system [13].

The calcineurin-NFAT signaling pathway is involved in the nerve activity-dependent regulation of muscle fiber-type-specific gene programs (reviewed in [14]), and one member of the NFAT family, NFATc1, acts as a slow-type nerve activity sensor in vivo $[15,16]$, whereas other members of the NFAT family are also responsive to fast-type nerve activity and might thus contribute to modulate the fast fiber phenotype [17]. An NFATc1-GFP fusion protein, when electroporated in skeletal muscles in vivo shows a predominantly cytoplasmic localization in the fast tibialis anterior muscle but a nuclear localization in the slow soleus. A rapid nuclear translocation of NFATc1 can be induced by electrical stimulation of tibialis anterior with an impulse pattern typical of slow motor neurons, while denervation causes a rapid nuclear export of NFATc1 in soleus [16]. NFAT nuclear translocation and transcriptional activity has been recently analyzed during the day-night cycle [7]. NFATc1-GFP shows an accumulation in mouse soleus myonuclei during the dark phase with a peak at Zeitgeber (ZT)16, and a similar circadian oscillation of luciferase activity is seen after electroporation of an NFAT-luciferase construct but with a 4-8-h delay. The NFAT target gene, Rcan1.4, shows a similar circadian oscillation with a peak during the dark phase, which is drastically

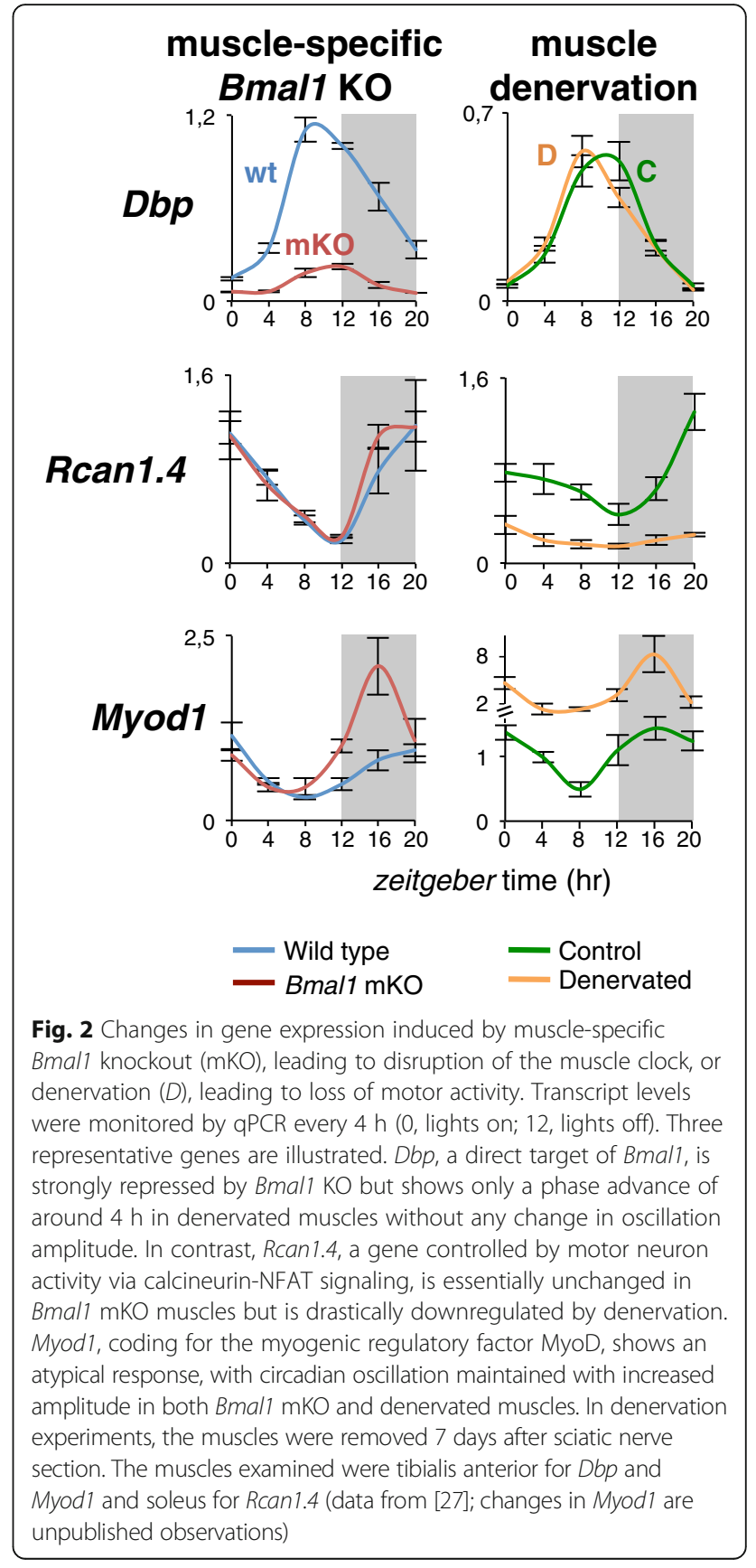

decreased in denervated muscle (Fig. 2). Interestingly, Rcan1.4 circadian oscillation is unchanged in musclespecific Bmal1 mKO muscles, supporting the notion that Rcan1.4 is a circadian muscle gene that is dependent on activity but independent of the core oscillator. It is possible that some of the changes induced by denervation may not reflect a direct effect of the loss of nerve activity but might be due to the transcriptional remodeling of gene expression that accompanies the denervation process. However, this possibility seems unlikely for NFAT target genes, as a 
drastic reduction of Rcan1.4 gene expression during the dark phase is already detected at $12 \mathrm{~h}$ after nerve section, before any significant change in transcriptional programs has taken place in denervated muscles [7].

\section{The role of the intrinsic muscle clock: lessons from Bmal1 knockout models}

The significance of the intrinsic muscle clock in muscle physiology has been addressed using genetic loss-offunction mouse models targeting the Bmall gene. BMAL1 is a crucial component of the molecular clock, and the only one for which loss results in complete disruption of circadian rhythms [18]. Different mouse lines have been generated to inactivate the Bmal1 gene in the skeletal muscles: as summarized in Table 1, these include both whole body and tissue-specific, constitutive, and inducible $\mathrm{KO}$ models.

Mice with whole body Bmal1 $\mathrm{KO}$, generated by standard methods leading to deletion of the gene in germinal cells, stop growing around 16 weeks of age, display progressive and dramatic muscle atrophy and decreased total activity level, and die between 26 and 52 weeks of age with signs of premature aging, including arthropathy, decreased hair growth, ocular abnormalities such as cataracts, and neurodegeneration with brain astrogliosis [18-20]. Bmal1 $\mathrm{KO}$ also causes altered metabolism, including altered response to insulin $[21,22]$ and ectopic fat accumulation in the skeletal muscle [23]. Some of these changes could be due to increased oxidative stress, since the loss of Bmal1 is known to cause accumulation of reactive oxygen species $[19,24]$ and antioxidant treatment was found to ameliorate symptoms of premature aging [25]. Muscle structure and function is altered in these mice even at early stages of postnatal development: at
12-14 weeks of age, muscle force is decreased, ultrastructural organization of thick and thin filament appears disrupted, and mitochondrial volume and respiratory function are decreased [26]. It was suggested that these changes are due to loss of function of the muscle-specific regulatory factor MyoD, because similar changes were found in Myod1 null mice and Myod1 was reported to be a target of BMAL1 and to lose its circadian oscillation in Bmal1 KO mice [26]. However, this interpretation is in contrast with a subsequent study on a muscle-specific Bmal1 KO model, obtained by crossing a mouse line bearing a floxed Bmall with an Mlc1f-Cre line, bearing Cre recombinase driven by the myosin light chain 1 fast promoter [27]. These mice show drastic reduction of Bmal1 transcripts in the skeletal muscle but not in the heart and other organs; however, they have normal life span and body weight with no obvious sign of premature aging. Muscle histology and ultrastructure are normal, and muscle weight is even increased with a slight decrease in normalized muscle force [27]. These findings suggest that the dramatic muscle atrophy found in whole body Bmal1 $\mathrm{KO}$ mice cannot result from a disrupted muscle clock or from loss of cell-autonomous function of Bmal1 in muscle fibers (see also [28]). In addition, Myod1 gene expression is increased rather than decreased in these mice and maintains its circadian oscillation with a peak during the dark phase of the cycle (Fig. 2). A similar effect, with an even greater upregulation of Myod1, is seen after denervation. Based on these results, it seems unlikely that MyoD can mediate the effect of BMAL1 function on the skeletal muscle, as previously suggested [26]. On the other hand, Myod1 gene expression is apparently controlled by feeding, as Myod 1 transcripts are strongly downregulated by fasting,

Table 1 Global phenotypes of different Bmal1 knockout models

\begin{tabular}{|c|c|c|c|c|c|c|}
\hline & Whole bod & al1 $\mathrm{KO}$ & & Muscle-sp & $B m a$ & \\
\hline & Standard & Standard + rescue with $\mathrm{mBmal1^{ \textrm {a } }}$ & $\overline{\text { Inducible }}$ & Standard & Indu & \\
\hline Circadian locomotor rhythm & No & No & No & Yes & Yes & Yes \\
\hline Total activity level & $\downarrow$ & $=$ & $=$ & $\uparrow$ & $=$ & $=$ \\
\hline Life span & $\downarrow$ & $=$ & $=$ & $=$ & $=$ & ND \\
\hline Body weight & $\downarrow$ & $=$ & $=$ & $=$ & $=$ & ND \\
\hline Muscle weight & $\downarrow$ & ND & $N D^{b}$ & $\uparrow$ & $=$ & ND \\
\hline Muscle fiber CSA & $\downarrow$ & ND & ND & $\uparrow$ & $=$ & $=$ \\
\hline Muscle force (normalized) & $\downarrow$ & ND & ND & $\downarrow$ & $=$ & $\downarrow$ \\
\hline Muscle fiber-type profile (fast muscles) & ND & ND & ND & $\downarrow 2 X$ & $=$ & $\downarrow 2 \mathrm{~B}$ \\
\hline References & {$[18,19,26]$} & [32] & {$[30]$} & {$[27]$} & {$[27]$} & {$[31,55]$} \\
\hline
\end{tabular}

ND not determined, $=$ unchanged, $\downarrow$ decreased, $\uparrow$ increased, CSA cross-sectional area

${ }^{a}$ Mice with muscle-specific rescue of Bmal1 null mice, obtained by crossing Bmal1 global KO mice with Bmal1 transgenic mice bearing a Bmal1 DNA construct driven by the muscle-specific a-actin promoter

${ }^{\mathrm{b}}$ Muscle weight was not determined but was likely unchanged because body weight and the weight of most organs, including fat deposits, were unchanged 
under conditions when Bmal1 transcripts maintain their normal levels and circadian pattern of expression [29].

Further insight into the function of Bmal1 in muscle fibers was obtained by inducible Bmal1 KO models. A ubiquitously inducible $\mathrm{KO}$ model was generated by crossing a floxed Bmal1 line with a tamoxifen-inducible universal Cre line [30]. Tamoxifen treatment was started in 3month-old mice leading to Bmal1 inactivation in all tissues at an adult stage, with the skeletal muscles showing a $99 \%$ reduction of Bmal1 mRNA levels at Zeitgeber time 0 (ZT0, lights on), when Bmal1 expression is high. These mice showed no significant difference in life span or body and organ weight when compared to control, suggesting that the dramatic phenotype seen in conventional Bmal1 $\mathrm{KO}$ mice results from BMAL1 function during development. Hair growth is normal, and there is no sign of agedependent arthropathy or calcification, although brain astrogliosis and ocular abnormalities, similar to those observed with prenatal Bmal1 KO [20], were also evident after postnatal Bmal1 depletion [30]. No difference was seen in glucose tolerance test (GTT) and insulin tolerance test (ITT) between $\mathrm{KO}$ and control mice. These results indicate that most phenotypes in conventional Bmal1 $\mathrm{KO}$ mice, previously attributed to disruption of circadian rhythms, reflect the loss of properties of BMAL1 during early development and are probably independent of its role in the clock (see below). However, BMAL1 appears to have a direct function in the eye and central nervous system irrespective of developmental issues and probably due to increased oxidative stress [30]. Muscle-specific inducible models were generated by crossing a floxed Bmal1 line with a tamoxifen-inducible Cre driven by the human $\alpha$-actin promoter, thus inducing Bmal1 inactivation exclusively in the skeletal muscle at an adult stage [27]. These mice have an essentially normal phenotype with respect to life span, body weight, and muscle mass (Table 1); however, they show altered glucose metabolism (see below). Muscle force and the proportion of type $2 \mathrm{~B}$ fibers were decreased in these mice, whereas fiber size was unchanged even at 12 months of age and centrally nucleated fibers were not detected [31].

Taken together, these studies suggest that the dramatic phenotype observed in the global Bmal1 KO, characterized by premature aging and death, with reduced body weight and muscle wasting, reflects the loss of Bmal1 function during development in non-muscle tissues. Two KO models support this conclusion. First, musclespecific Bmal1 KO, leading to Bmal1 deletion since early developmental stages selectively in the skeletal muscle, does not induce significant changes with the exception of altered muscle metabolism [27], pointing to a major effect of Bmal1 in non-muscle tissues in the pathogenesis of sarcopenia, premature aging, and reduced life span. Second, these changes are also absent when Bmal1 $\mathrm{KO}$ is induced ubiquitously at an adult stage, therefore must reflect the loss of Bmal1 during development [30].

However, another experimental model seems to contradict this interpretation. Mice with muscle-specific rescue of Bmal1 null mice, obtained by crossing Bmal1 global $\mathrm{KO}$ mice with Bmal1 transgenic mice bearing a Bmal1 DNA construct driven by the muscle-specific human $\alpha$-actin promoter, show normal body weight and total activity level, as well as longer life span compared to Bmal1 null mice [32]. Although incomplete information was provided on some critical aspects (life span profile, muscle weight), which does not allow a thorough evaluation of this model, some considerations could be put forward to explain this surprising result. A number of studies in Drosophila have revealed that the skeletal muscle can affect the global body phenotype (reviewed in [33, 34]). This conclusion is supported by studies in mammals, for example, reduced body size, but essentially normal skeletal muscle phenotype, is observed when myogenin, a muscle-specific transcription factor, is deleted at birth using an inducible KO model [35]. Some effects of the skeletal muscle on the whole organism can be mediated by the release of secreted factors from muscle cells. Hundreds of muscle genes, potentially coding for secreted proteins, are expressed in skeletal muscle [36], and specific myokines have been identified (reviewed in [37]). Myokines can transmit signals from the muscles to adipose tissue or other organs and, in this way, affect global aspects of the phenotype (see $[33,34])$. Interestingly, the secretion of several myokines is strongly downregulated by siRNA targeting Clock in skeletal myotubes, showing that the muscle clock is involved in the regulation of basal myokine secretion [38]. One wonders whether the muscle Bmal1dependent rescue of the phenotype of Bmal1 null mice is mediated by a myokine release mechanism. Bmal1, like other core clock genes, is expressed since early embryonic stages in both the SCN and peripheral tissues but does not oscillate during embryogenesis (Fig. 3), clock rhythm being established during the first week after birth [39]. The role of Bmal1 in the embryo is not known, but one possibility is that this gene might have specific non-clock-related functions, for which loss might account for premature aging [30]. Indeed, a recent study indicates that BMAL1 stimulates translation independently of its transcriptional activity. The ribosomal S6 protein kinase 1 (S6K1), an important mTOR-dependent regulator of translation, rhythmically phosphorylates BMAL1, which in turn interacts with the translational machinery, promoting protein synthesis [40].

\section{Role of the muscle clock in glucose uptake and metabolism}

Glucose metabolism is altered by disruption of circadian rhythms induced by SCN lesion or global inactivation of clock genes (see [41]). However, both types of 


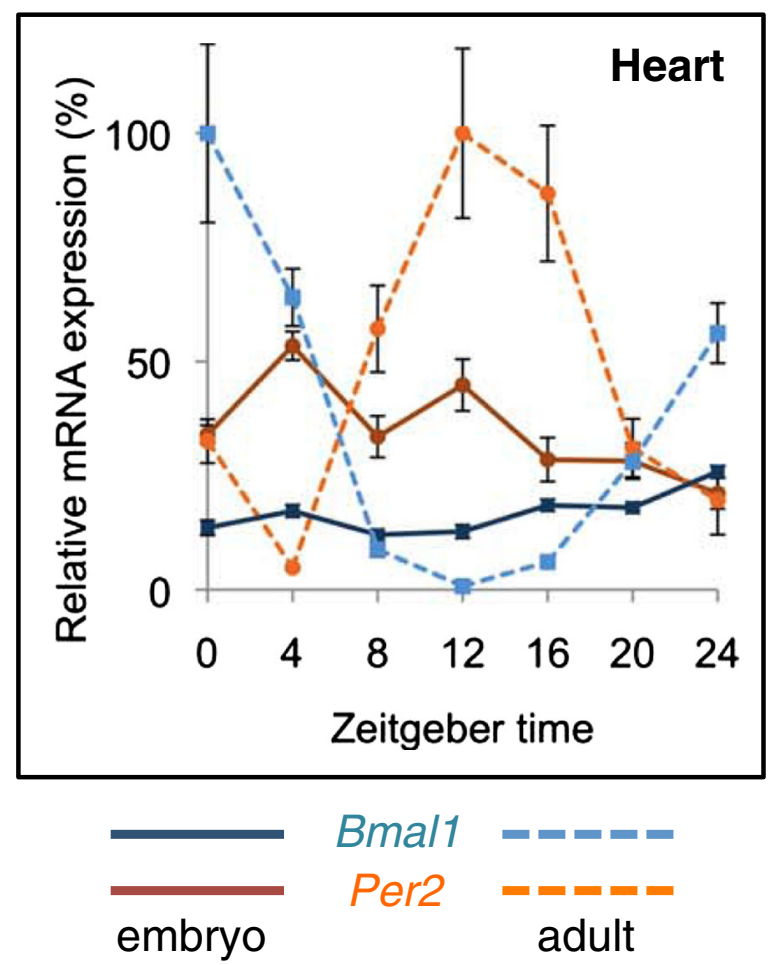

Fig. 3 Bmal1 and Per2 transcripts do not oscillate in embryonic tissues. Twenty-four-hour expression profiles of Per2 and Bmal1 mRNA in embryonic (E18-E19) and adult mouse heart, as determined by $\mathrm{qPCR}$. Identical results were seen in the liver and kidney, while the skeletal muscle was not analyzed in this study. Note that the embryonic heart shows little circadian variation in Per2 and Bmal1 expression, in contrast with the robust changes seen in the adult tissue (modified from [39])

interventions profoundly affect behavior, including locomotor activity and feeding rhythms, and may thus indirectly alter metabolism. Tissue-specific Bmal1 KO models allow to identify the direct role of local clock mechanisms in glucose metabolism in the presence of normal locomotor activity and feeding rhythms. These models have revealed the crucial contribution of peripheral clocks in the pancreas, liver, and skeletal muscle on glucose metabolism (Fig. 4). Liver-specific ablation of Bmal1 causes hypoglycemia during the fasting phase, when plasma glucose is controlled by the liver through glycogenolysis and gluconeogenesis, due to loss of rhythmic expression of hepatic glucose regulatory genes, responsible for hepatic glucose production and export [42]. Selective inactivation of Bmal1 in the pancreas or in the $\beta$ cells causes hyperglycemia due to impaired insulin secretion [43-45], and isolated pancreatic islets from adult mice display circadian oscillation in glucosestimulated insulin secretion which is abrogated by Bmal1 ablation [46]. Transcriptome analyses showed that a large number of genes involved in insulin secretion display circadian changes in expression and

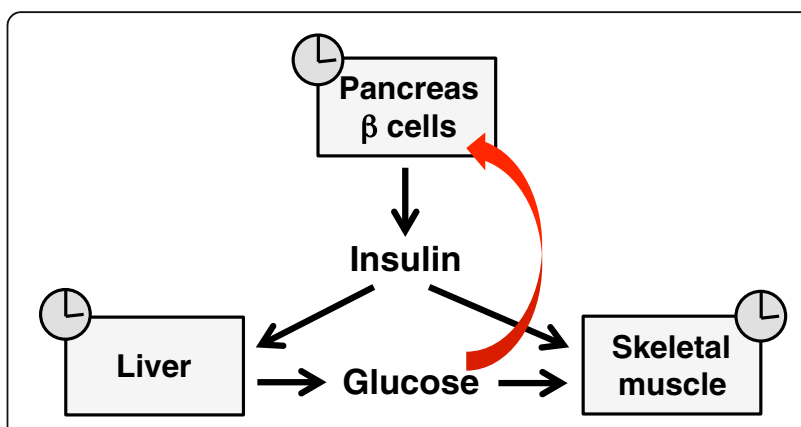

Fig. 4 The scheme illustrates the role of peripheral clocks in the control of muscle glucose metabolism, as determined using tissue-specific Bmal1 knockout models. The liver clock controls glucose output during the fasting/inactive phase, as shown by the finding that liver-specific Bmal1 $\mathrm{KO}$ causes hypoglycemia during this phase [42]. The pancreas $\beta$ cell clock controls insulin secretion, as $\beta$ cell-specific Bmal1 KO causes hyperglycemia [43-45]. The muscle clock promotes glucose uptake and metabolism at awakening, as skeletal muscle-specific Bmal1 KO causes impaired insulin-dependent glucose uptake and glucose oxidation in skeletal muscle fibers [27]

chromatin immunoprecipitation revealed that CLOCK and BMAL1 control cycling genes in $\beta$ cells by binding at distal regulatory elements. Severe glucose intolerance is induced in adult mice using a tamoxifen-inducible model of pancreas-specific Bmal1 knockout [46]. On the other hand, glucose metabolism in adipose tissue is apparently unaffected by the local clock, as adipose tissue of mice with adipocyte-specific $\mathrm{KO}$ of Bmal1 shows normal insulin sensitivity compared to control littermates despite the greater body adiposity [47].

The skeletal muscle is the predominant site of insulinstimulated glucose disposal in the postprandial state [48], and muscle insulin resistance is one of the earliest factors in the pathogenesis of the metabolic syndrome $[49,50]$. Dyar et al. [27], using both constitutive and inducible muscle-specific Bmal1 KO models, revealed that the muscle clock controls glucose uptake and metabolism. This conclusion was based on studies of gene expression at the transcript and protein level, integrated by enzymatic assays, glucose uptake studies, and circadian metabolomics analyses. Insulin-stimulated glucose uptake is impaired in the skeletal muscles from musclespecific Bmal1 KO mice, likely due to reduced transcript and protein levels of glucose transporter 4 (GLUT4), the insulin-dependent glucose transporter (Fig. 5). GLUT4 protein levels were reduced by $45 \%$ across the diurnal cycle, a change similar to that seen in heterozygous GLUT4 $^{+/-}$mice, which also show a reduced insulinstimulated glucose uptake in the skeletal muscles [51]. Muscle-specific Bmal1 KO mice are also characterized by reduced transcript and protein levels of TBC1D1, a Rab-GTPase involved in GLUT4 translocation to the plasma membrane. Tbc1d1 KO mice likewise show a 50 \% reduction in GLUT4 levels and impaired insulin- 


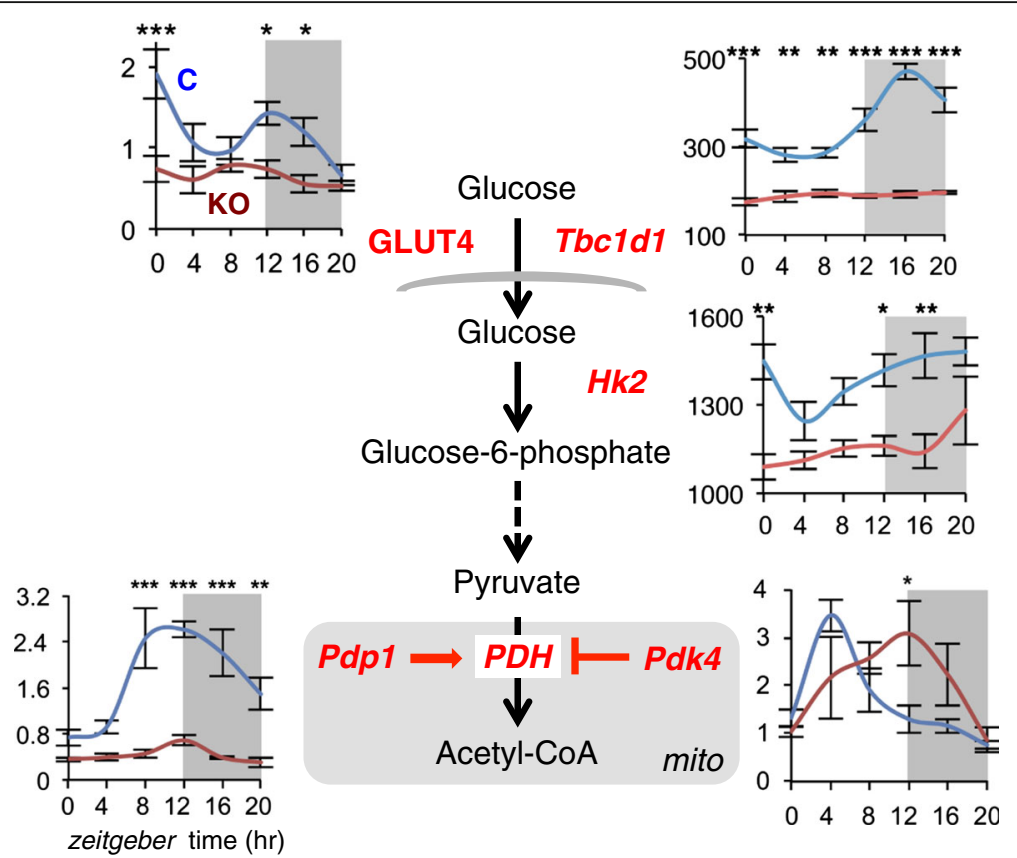

Fig. 5 Simplified scheme of glucose uptake and metabolism in muscle cells, highlighting two crucial steps controlled by the intrinsic muscle clock: insulin-dependent glucose uptake and pyruvate conversion to acetyl-CoA. Insulin promotes glucose uptake by activating the kinase AKT that phosphorylates the Rab-GTPase-activating protein TBC1D1, thus promoting the translocation of GLUT4 to the plasma membrane. Pyruvate, upon entry into mitochondria (mito), is metabolized to acetyl-CoA by pyruvate dehydrogenase (PDH), whose activity is inhibited by the PDH kinase PDK4 and stimulated by the PDH phosphatase PDP1. The protein expression of GLUT4, and both mRNA and protein levels of TBC1D1, PDK4, and PDP1 vary across the day-night cycle (0, lights on; 12, lights off) and are drastically affected by Bmal1 mKO. Under normal conditions, PDK4 has a peak of expression in the fasting phase (around ZT4), whereas PDP1 peaks around the transition from the fasting to the feeding/active phase (around ZT12). Note that PDK4 starts to decrease and PDP1 to increase during the fasting phase, before awakening, supporting the notion of the anticipatory role of the muscle clock, which prepares the muscles to the upcoming activity period. These circadian adaptations are completely disrupted by Bmal1 mKO, with downregulation of PDP1 and a rightward shift in the peak of PDK4, leading to decrease in PDH activity at awakening (data from [27])

stimulated glucose uptake in the skeletal muscle [52]; therefore, it is likely that the loss of BMAL1 in the skeletal muscle causes decreased TBC1D1 levels which in turn leads to reduced GLUT4 and impaired insulinstimulated glucose uptake. TBC1D1 has a circadian oscillation with a peak in the active/feeding phase but starts to increase already in the late fasting phase. In contrast, the expression of another Rab-GTPase involved in GLUT4 translocation, TBC1D4, shows no circadian oscillation in the skeletal muscle and is unaffected by Bmal1 $\mathrm{KO}$ [27]. The phosphorylation of glucose to glucose-6phosphate by hexokinase is also affected by the muscle clock, as suggested by the significantly reduced levels of hexokinase 2 (HK2) after Bmal1 inactivation. Pyruvate dehydrogenase (PDH) activity is reduced in the skeletal muscles due to altered expression of circadian genes Pdk4 and Pdp1, coding for PDH kinase and phosphatase, respectively. Under normal conditions, PDH kinase 4 (PDK4), which inhibits PDH activity, shows a peak of expression during the fasting phase (around ZT4), whereas PDH phosphatase 1 (PDP1), which stimulates PDH activity, peaks around the transition from the fasting to the feeding phase (around
ZT12). Interestingly, PDK4 starts to decrease whereas PDP1 begins to increase during the fasting phase, before awakening, supporting the notion of the anticipatory role of the muscle clock, which prepares the muscles for the upcoming activity period. Accordingly, muscle clock disruption leads to $\mathrm{PDH}$ inhibition, thus uncoupling glycolysis from glucose oxidation, and contributing to the diversion of glycolytic intermediates to alternative metabolic pathways, as revealed by metabolomics analysis [27].

The tissue-specific role of BMAL1 in regulating insulin sensitivity in muscle cells is supported by studies on cultured $\mathrm{C} 2 \mathrm{C} 12$ myotubes [53]. In these cells, insulin sensitivity is reduced by knockdown of Clock or Bmal1, while the insulin resistance induced by palmitate is improved by Clock and Bmal1 overexpression [53]. These effects appear to be mediated by SIRT1, which is a target of CLOCK and BMAL1, as shown by the finding that (i) Sirt1 knockdown blocks the improvement induced by Clock and Bmal1 overexpression on the palmitatedependent insulin resistance and (ii) Sirt1 overexpression ameliorates insulin resistance induced by knockdown of Clock or Bmal1. 


\section{Conclusions}

The comparative analysis summarized in Table 1 shows that the muscle phenotype is variably affected in different Bmal1 KO models. In particular, the dramatic muscle wasting and premature aging found in the global conventional $\mathrm{KO}$ are not present in muscle-specific Bmal1 $\mathrm{KO}$ or in global $\mathrm{KO}$ induced in the adult, thus must reflect the loss of Bmal1 function during development and in non-muscle tissues. These findings indicate that the intrinsic muscle clock is dispensable for muscle growth and that its inactivation does not cause premature aging and reduced life span. The fact that core clock genes are not oscillating in embryonic tissues points to possible non-clock functions of Bmal1 during development, such as the recently identified BMAL1 role in the control of protein synthesis. On the other hand, Bmal1 circadian oscillation in the skeletal muscle is involved in adult muscle metabolism. In particular, two models of muscle-specific inactivation of Bmal1 suggest that the intrinsic muscle clock controls both glucose uptake and glucose metabolism in the skeletal muscle and support the conclusion that "a major physiological role of the muscle clock is to prepare the tissue for the transition from the rest/fasting phase to the active/feeding phase, when glucose becomes the predominant fuel for skeletal muscle" [27]. It will be important to confirm this conclusion with muscle-specific $\mathrm{KO}$ of other clock genes, e.g., double KO of Per1 and Per2, or Cry 1 and Cry2, in order to establish unambiguously that the changes in muscle metabolism induced by Bmal1 $\mathrm{KO}$ result from the disruption of the muscle clock and not from specific functions of Bmal1.

\section{Note added in proof}

After submission of our manuscript we became aware of a recent study describing another muscle-specific Bmal1 KO model obtained by crossing floxed Bmal1 with MCK-Cre mice [54]. These mice have a normal life span, thus confirming the results of Dyar et al. [27], and show a denervation-induced increased in Myod1 expression similar to that of wild type mice.

\section{Abbreviations}

CK2: Casein kinase 2; GLUT4: Glucose transporter 4; GTT: Glucose tolerance test; HK2: Hexokinase 2; ITT: Insulin tolerance test; KO: Knockout; PDH: Pyruvate dehydrogenase; PDK4: PDH kinase 4; PDP1: PDH phosphatase 1; SCN: Suprachiasmatic nucleus; ZT: Zeitgeber

\section{Acknowledgements}

Not applicable

\section{Funding}

Not applicable

Availability of data and materials

Not applicable
Authors' contributions

$\mathrm{SS}, \mathrm{BB}$, and $\mathrm{KAD}$ contributed to the writing and editing of this manuscript. All authors read and approved the manuscript.

\section{Competing interests}

The authors declare that they have no competing interests.

\section{Consent for publication}

Not applicable

\section{Ethics approval and consent to participate}

Not applicable

\section{Author details}

${ }^{1}$ Venetian Institute of Molecular Medicine (VIMM), Via Orus 2, 35129 Padova, Italy. ${ }^{2}$ Department of Biomedical Sciences, University of Padova, Padova, Italy. ${ }^{3}$ Molecular Endocrinology, Institute for Diabetes and Obesity, Helmholtz Zentrum München, Munich, Germany.

Received: 4 August 2016 Accepted: 28 September 2016

Published online: 13 October 2016

\section{References}

1. Reppert SM, Weaver DR. Coordination of circadian timing in mammals. Nature. 2002;418:935-41.

2. Dibner $C$, Schibler $U$, Albrecht $U$. The mammalian circadian timing system: organization and coordination of central and peripheral clocks. Annu Rev Physiol. 2010;72:517-49.

3. Tamaru T, Hattori M, Honda K, Nakahata $Y$, Sassone-Corsi $P$, van der Horst GT, et al. CRY drives cyclic CK2-mediated BMAL1 phosphorylation to control the mammalian circadian clock. PLoS Biol. 2015:13:e1002293.

4. Yu EA, Weaver DR. Disrupting the circadian clock: gene-specific effects on aging, cancer, and other phenotypes. Aging (Albany NY). 2011;3:479-93.

5. Kornmann B, Schaad O, Bujard H, Takahashi JS, Schibler U. System-driven and oscillator-dependent circadian transcription in mice with a conditionally active liver clock. PLoS Biol. 2007;5:e34.

6. Damiola F, Le Minh N, Preitner N, Kornmann B, Fleury-Olela F, Schibler U. Restricted feeding uncouples circadian oscillators in peripheral tissues from the central pacemaker in the suprachiasmatic nucleus. Genes Dev. 2000;14: 2950-61.

7. Dyar KA, Ciciliot S, Tagliazucchi GM, Pallafacchina G, Tothova J, Argentini C, et al. The calcineurin-NFAT pathway controls activity-dependent circadian gene expression in slow skeletal muscle. Mol Metab. 2015;4:823-33.

8. Miller BH, McDearmon EL, Panda S, Hayes KR, Zhang J, Andrews JL, et al. Circadian and CLOCK-controlled regulation of the mouse transcriptome and cell proliferation. Proc Natl Acad Sci U S A. 2007;104:3342-47.

9. Mayeuf-Louchart A, Staels B, Duez H. Skeletal muscle functions around the clock. Diabetes Obes Metab. 2015;17 Suppl 1:39-46.

10. Wolff $\mathrm{G}$, Esser KA. Scheduled exercise phase shifts the circadian clock in skeletal muscle. Med Sci Sports Exerc. 2012:44:1663-70.

11. Zambon AC, McDearmon EL, Salomonis N, Vranizan KM, Johansen KL, Adey $\mathrm{D}$, et al. Time- and exercise-dependent gene regulation in human skeletal muscle. Genome Biol. 2003:4:R61.

12. Hennig R, Lomo T. Firing patterns of motor units in normal rats. Nature. 1985:314:164-6.

13. Nakao R, Yamamoto S, Horikawa K, Yasumoto Y, Nikawa T, Mukai C, et al. Atypical expression of circadian clock genes in denervated mouse skeletal muscle. Chronobiol Int. 2015:32:486-96.

14. Schiaffino S, Serrano A. Calcineurin signaling and neural control of skeletal muscle fiber type and size. Trends Pharmacol Sci. 2002;23:569-75.

15. McCullagh KJ, Calabria E, Pallafacchina G, Ciciliot S, Serrano AL, Argentini C, et al. NFAT is a nerve activity sensor in skeletal muscle and controls activitydependent myosin switching. Proc Natl Acad Sci U S A. 2004;101:10590-95.

16. Tothova J, Blaauw B, Pallafacchina G, Rudolf R, Argentini C, Reggiani C, et al. NFATC1 nucleocytoplasmic shuttling is controlled by nerve activity in skeletal muscle. J Cell Sci. 2006;119:1604-11.

17. Calabria E, Ciciliot S, Moretti I, Garcia M, Picard A, Dyar KA, et al. NFAT isoforms control activity-dependent muscle fiber type specification. Proc Natl Acad Sci U S A. 2009;106:13335-40. 
18. Bunger MK, Wilsbacher LD, Moran SM, Clendenin C, Radcliffe LA, Hogenesch $\mathrm{JB}$, et al. Mop3 is an essential component of the master circadian pacemaker in mammals. Cell. 2000;103:1009-17.

19. Kondratov RV, Kondratova AA, Gorbacheva VY, Vykhovanets OV, Antoch MP. Early aging and age-related pathologies in mice deficient in BMAL1, the core component of the circadian clock. Genes Dev. 2006;20:1868-73.

20. Musiek ES, Lim MM, Yang G, Bauer AQ, Qi L, Lee Y, et al. Circadian clock proteins regulate neuronal redox homeostasis and neurodegeneration. J Clin Invest. 2013;123:5389-400.

21. Rudic RD, McNamara P, Curtis AM, Boston RC, Panda S, Hogenesch JB, et al. BMAL1 and CLOCK, two essential components of the circadian clock, are involved in glucose homeostasis. PLoS Biol. 2004;2:e377.

22. Shi SQ, Ansari TS, McGuinness OP, Wasserman DH, Johnson CH. Circadian disruption leads to insulin resistance and obesity. Curr Biol. 2013;23:372-81.

23. Shimba S, Ogawa T, Hitosugi S, Ichihashi Y, Nakadaira Y, Kobayashi M, et al. Deficient of a clock gene, brain and muscle Arnt-like protein-1 (BMAL1), induces dyslipidemia and ectopic fat formation. PLoS One. 2011;6:e25231.

24. Khapre RV, Kondratova AA, Susova O, Kondratov RV. Circadian clock protein BMAL1 regulates cellular senescence in vivo. Cell Cycle. 2011;10:4162-9.

25. Kondratov RV, Vykhovanets O, Kondratova AA, Antoch MP. Antioxidant N-acetylL-cysteine ameliorates symptoms of premature aging associated with the deficiency of the circadian protein BMAL1. Aging (Albany NY). 2009;1:979-87.

26. Andrews JL, Zhang X, McCarthy JJ, McDearmon EL, Hornberger TA, Russell $B$, et al. CLOCK and BMAL1 regulate MyoD and are necessary for maintenance of skeletal muscle phenotype and function. Proc Natl Acad Sci U S A. 2010;107:19090-5.

27. Dyar KA, Ciciliot S, Wright LE, Bienso RS, Tagliazucchi GM, Patel VR, et al. Muscle insulin sensitivity and glucose metabolism are controlled by the intrinsic muscle clock. Mol Metab. 2014;3:29-41.

28. Dyar KA, Schiaffino S, Blaauw B. Inactivation of the intrinsic muscle clock does not cause sarcopenia. J Physiol. 2016;594:3161-2.

29. Shavlakadze T, Anwari T, Soffe Z, Cozens G, Mark PJ, Gondro C, et al. Impact of fasting on the rhythmic expression of myogenic and metabolic factors in skeletal muscle of adult mice. Am J Physiol Cell Physiol. 2013;305:C26-35.

30. Yang G, Chen L, Grant GR, Paschos G, Song WL, Musiek ES, et al. Timing of expression of the core clock gene Bmal1 influences its effects on aging and survival. Sci Transl Med. 2016;8:324ra316.

31. Schroder EA, Harfmann BD, Zhang X, Srikuea R, England JH, Hodge BA, et al. Intrinsic muscle clock is necessary for musculoskeletal health. J Physiol. 2015;593:5387-404.

32. McDearmon EL, Patel KN, Ko CH, Walisser JA, Schook AC, Chong JL, et al. Dissecting the functions of the mammalian clock protein BMAL1 by tissuespecific rescue in mice. Science. 2006;314:1304-8.

33. Owusu-Ansah E, Perrimon N. Stress signaling between organs in metazoa. Annu Rev Cell Dev Biol. 2015:31:497-522.

34. Rai M, Demontis F. Systemic nutrient and stress signaling via myokines and myometabolites. Annu Rev Physiol. 2016;78:85-107.

35. Knapp JR, Davie JK, Myer A, Meadows E, Olson EN, Klein WH. Loss of myogenin in postnatal life leads to normal skeletal muscle but reduced body size. Development. 2006;133:601-10

36. Bortoluzzi S, Scannapieco P, Cestaro A, Danieli GA, Schiaffino S. Computational reconstruction of the human skeletal muscle secretome. Proteins. 2006;62:776-92.

37. Pedersen BK, Febbraio MA. Muscles, exercise and obesity: skeletal muscle as a secretory organ. Nat Rev Endocrinol. 2012;8:457-65.

38. Perrin L, Loizides-Mangold U, Skarupelova S, Pulimeno P, Chanon S, Robert $M$, et al. Human skeletal myotubes display a cell-autonomous circadian clock implicated in basal myokine secretion. Mol Metab. 2015;4:834-45.

39. Dolatshad H, Cary AJ, Davis FC. Differential expression of the circadian clock in maternal and embryonic tissues of mice. PLoS One. 2010;5:e9855.

40. Lipton JO, Yuan ED, Boyle LM, Ebrahimi-Fakhari D, Kwiatkowski E, Nathan A, et al. The circadian protein BMAL1 regulates translation in response to S6K1-mediated phosphorylation. Cell. 2015;161:1138-51.

41. Kalsbeek A, la Fleur S, Fliers E. Circadian control of glucose metabolism. Mol Metab. 2014;3:372-83.

42. Lamia KA, Storch KF, Weitz CJ. Physiological significance of a peripheral tissue circadian clock. Proc Natl Acad Sci U S A. 2008;105:15172-7.

43. Lee J, Moulik M, Fang Z, Saha P, Zou F, Xu Y, et al. Bmal1 and beta-cell clock are required for adaptation to circadian disruption, and their loss of function leads to oxidative stress-induced beta-cell failure in mice. Mol Cell Biol. 2013;33:2327-38.
44. Marcheva B, Ramsey KM, Buhr ED, Kobayashi Y, Su H, Ko CH, et al. Disruption of the clock components CLOCK and BMAL1 leads to hypoinsulinaemia and diabetes. Nature. 2010;466:627-31.

45. Sadacca LA, Lamia KA, deLemos AS, Blum B, Weitz CJ. An intrinsic circadian clock of the pancreas is required for normal insulin release and glucose homeostasis in mice. Diabetologia. 2011;54:120-4.

46. Perelis M, Marcheva B, Ramsey KM, Schipma MJ, Hutchison AL, Taguchi A, et al. Pancreatic beta cell enhancers regulate rhythmic transcription of genes controlling insulin secretion. Science. 2015;350:aac4250.

47. Paschos GK, Ibrahim S, Song WL, Kunieda T, Grant G, Reyes TM, et al. Obesity in mice with adipocyte-specific deletion of clock component Arntl. Nat Med. 2012;18:1768-77.

48. DeFronzo RA, Jacot E, Jequier E, Maeder E, Wahren J, Felber JP. The effect of insulin on the disposal of intravenous glucose. Results from indirect calorimetry and hepatic and femoral venous catheterization. Diabetes. 1981; 30:1000-7.

49. DeFronzo RA, Tripathy D. Skeletal muscle insulin resistance is the primary defect in type 2 diabetes. Diabetes Care. 2009;32 Suppl 2:S157-163.

50. Rabol R, Petersen KF, Dufour S, Flannery C, Shulman Gl. Reversal of muscle insulin resistance with exercise reduces postprandial hepatic de novo lipogenesis in insulin resistant individuals. Proc Natl Acad Sci U S A. 2011; 108:13705-9.

51. Zisman A, Peroni OD, Abel ED, Michael MD, Mauvais-Jarvis F, Lowell BB, et al. Targeted disruption of the glucose transporter 4 selectively in muscle causes insulin resistance and glucose intolerance. Nat Med. 2000;6:924-8.

52. Dokas J, Chadt A, Nolden T, Himmelbauer H, Zierath JR, Joost HG, et al. Conventional knockout of Tbc1d1 in mice impairs insulin- and AICARstimulated glucose uptake in skeletal muscle. Endocrinology. 2013;154: 3502-14.

53. Liu J, Zhou B, Yan M, Huang R, Wang Y, He Z, et al. CLOCK and BMAL1 regulate muscle insulin sensitivity via SIRT1 in male mice. Endocrinology. 2016;157:2259-69.

54 Nakao R, Shimba S, Oishi K. Muscle Bmal1 is dispensable for the progress of neurogenic muscle atrophy in mice. J Circad Rhythms. 2016;14:1-7.

55. Hodge BA, Wen Y, Riley LA, Zhang X, England JH, Harfmann BD, et al. The endogenous molecular clock orchestrates the temporal separation of substrate metabolism in skeletal muscle. Skelet Muscle. 2015:5:17.

\section{Submit your next manuscript to BioMed Central and we will help you at every step:}

- We accept pre-submission inquiries

- Our selector tool helps you to find the most relevant journal

- We provide round the clock customer support

- Convenient online submission

- Thorough peer review

- Inclusion in PubMed and all major indexing services

- Maximum visibility for your research

Submit your manuscript at www.biomedcentral.com/submit
) Biomed Central 